\title{
A Resumption Strategy for Gapless Multiple Right Dislocation*
}

\author{
Jeong-Shik Lee \\ (Wonkwang University)
}

\begin{abstract}
Lee, Jeong-Shik. (2017). A Resumption Strategy for Gapless Multiple Right Dislocation. Language Research, 53.1, 23-58.
\end{abstract}

This paper mainly focuses on gapless multiple right dislocation constructions in Korean. Abe (2016) argues against the most popular approach to right dislocation, namely, a bi-clausal analysis incorporating repair by ellipsis strategy. Instead, he argues for a bi-clausal in situ deletion approach based on island insensitivity, intervention effects, and multiple sluicing in Japanese. In this paper, I carefully examine Abe's arguments, using corresponding Korean examples, and argue that his arguments are not tenable. I even claim that the bi-clausal analysis is not an option. More specifically, I propose that the in situ deletion effects are achieved by another mechanism, which I call phonetic resumption, under the mono-clausal analysis coupled with TP-raising.

Keywords: multiple right dislocation, gapless, in situ deletion, bi-clausal, mono-clausal, repair by ellipsis, phonetic resumption, TP-raising

\section{Introduction}

There has been a lot of discussion on right dislocation phenomena in Korean. Among different types of right dislocation (henceforth, $\mathrm{RD}$ ), this paper focuses on gapless multiple $\mathrm{RD}$ constructions (RDCs) that Abe (2016) provides from Japanese. In the ensuing discussion, I use corresponding Korean examples. One example of this type is provided below.

\footnotetext{
* I thank three anonymous reviewers for their critical comments on this paper. Any remaining flaws and errors, however, are solely mine.
} 
(1) John-i Barriers-lul ilk-ess-e, John-i Barriers-lul. John-Nom Barriers-Acc read-Past-Dec John-Nom Barriers-Acc 'Lit. John read Barriers, John Barriers.'

(Abe 2016, (1))

The above multiple gapless RDC results from rightward repetition of the matching elements in the first clause as $\mathrm{RD}$ remnants.

The most popular approach to $\mathrm{RD}$ is a bi-clausal analysis incorporating Move \& Delete in obtaining RD elements (Tanaka 2001 for Japanese, Chung 2009 for Korean). Thus the structure (1) is derived, as illustrated below.

(2) [TP John-i Barriers-lul ilk-ess-e], [TP John-i [TP Barriers-lul [TP <John-i> <Barriers-lul> ilk-ess-e] ] (Abe 2016, (2))

Two remnant movements of John-i and Barriers-lul are assumed in the second clause and the deletion of the following clausal constituent under identity with the preceding clause has applied except the remnants.

Abe (2016), however, argues against the repair by ellipsis strategy and instead argues for the in situ deletion approach based on island insensitivity, intervention effects, and multiple sluicing in Japanese. Under this approach, (1) has the following structure:

(3) [TP John-i Barriers-lul ilk-ess-e], [TP John-i Barriers-lul ilk-ess-e] [Focus] [Focus] (Abe 2016, (7))

In this paper, I carefully examine Abe's arguments, using corresponding Korean examples, and show that his arguments are not tenable. I even claim that the bi-clausal analysis is not an option; more specifically, I propose that the in situ deletion effects are achieved by another strategy, which I call phonetic resumption, under the mono-clausal analysis. While dealing with gapless RDCs, I also discuss gappy RDCs and pursue a uniform mono-clausal analysis to both types of RDC. 


\section{Abe's (2016) in situ Approach}

Abe (2016) points out that the bi-clausal approach introduced in (2) is problematic in dealing with island sensitivity. This is based on the following contrast between gapless RDCs in (4) and gappy RDCs in (5) with respect to island sensitivity.

(4) a. Mary-ka [John-i Barriers-lul ilk-un] hay-lul kiekha-ess-e, Mary-Nom John-Nom Barriers-Acc read year-Acc remembered, Barriers-lul.

Barriers-Acc

(Abe 2016, (3a))

'Lit. Mary remembered the year when John read Barriers, Barriers.'

b. Mary-ka [John-i Barriers-lul ilk-ess-ki ttaymwuney nolla-ess-e, Mary-Nom John-Nom Barriers-Acc read because was.surprised Barriers-lul.

Barriers-Acc

(Abe 2016, (3b))

'Lit. Mary was surprised because John read Barriers, Barriers.'

(5) a. ?*Mary-ka [John-i e ilk-un] hay-lul kiekha-ess-e,

Mary-Nom John-Nom read year-Acc remembered,

Barriers-lul.

Barriers-Acc

(Abe 2016, (4a))

'Lit. Mary remembered the year when John read $e_{1}$, Barriers.'.

b. ?*Mary-ka [John-i e ilk-ess-ki ttaymwuney nolla-ess-e,

Mary-Nom John-Nom read because was.surprised

Barriers-lul.

Barriers-Acc

(Abe 2016, (4b))

'Lit. Mary was surprised because John read $e_{1}$, Barriers.'.

Although the island effects are repaired by ellipsis in (4), the effects are not cancelled by ellipsis in (5). The movement of the remnant out of islands is therefore wrongly allowed in the case of gappy RDC in (5).

For this contrast, Abe (2016) proposes that while the gapless RDC in (4a) involves no movement, as illustrated in (6a), hence exhibiting no island effects, the gappy RDC in (5a) involves movement of the remnant, as seen in (6b), hence exhibiting island effects (only (4a) and (5a) are taken to avoid redundancy). 
(6) a. Mary-ka [John-i Barriers-lul ilk-un] hay-lul kiekha-ess-e, Mary-ka [John-i Barriers-lul ilk-un] hay-lul kiekha-ess-e.

b. ?*Mary-ka [John-i e ilk-un] hay-lul kiekha-ess-e, Barriers-lul $1_{1}$ John-i $t_{1}$-ilk-un] hay-lul kiekha-ess-e]

Here two questions arise as to why in situ deletion applies to the gapless $\mathrm{RDC}$ in (6a) and why the movement of the remnant is required in the gappy RDC in (6b). Abe (2016: 6) assumes that the second occurrence of Barriers-lul in (4a) bears a [Focus] feature and that deletion applies to a given constituent in such a way that all the materials except a phrase bearing a [Focus] feature get deleted, as seen in (6a).1) In this case, as an answer to the first question, he (p. 6) further assumes that the relevant identity condition on deletion does not require the phrase bearing a [Focus] feature to move out of the ellipsis site, since both the first and the second clauses in (6a) are simply identical. As for the second question, Abe (2016: 6) assumes that differently from (6a), the movement of Barriers-lul is required in (6b) to create a variable that can be plausibly regraded as non-distinct from the corresponding unspecified null object in the first clause.

My simple paraphrase of Abe's assumptions is as follows. In the gapless $\mathrm{RDC}$ in (6a), the RD element does not move since the two clauses are strictly identical/parallel, with no gap in the first clause; in the gappy $\mathrm{RDC}$ in (6b), the RD element must move to create a variable, which now can be identical/parallel to the gap in the first clause. $\left.{ }^{2}\right)$

Now, under Abe's in situ analysis of the gapless type of multiple $\mathrm{RD}$, (1) has the following structure, repeated below:

1) Abe (2015) actually uses a simpler example (his (6)) than (6a), but the relevant point remains the same. Also, notice that Abe allows non-constituent deletion in deriving the $\mathrm{RD}$ element here. This point will be brought up again later.

2) If the remnant movement in (6b) leaves a usual copy $<$ Barriers-lul $>$ in the variable position in the second clause, it becomes unclear whether the identity/parallelism between the two clauses can be satisfied in a strict sense.

(i) ?*Mary-ka [John-i e ilk-un] hay-lul kiekha-ess-e, Barriers-lul [John-i <Barriers-lul> ilk-un] hay-lul kiekha-ess-e]

I will ignore this issue in the ensuing discussion. 
(3) [TP John-i Barriers-lul ilk-ess-e], [TP John-i Barriers-lul ilk-ess-e]

[Focus] [Focus] (Abe 2016, (7))

Thus it is predicted that gapless multiple $\mathrm{RD}$ displays no island effects in the contexts of $(3 a, b)$. This prediction is borne out:

(7) a. Mary-ka [John-i Barriers-lul ilk-un] hay-lul kiekha-ess-e, Mary-Nom John-Nom Barriers-Acc read year-Acc remembered, John-i Barriers-lul.

John-Nom Barriers-Acc (Abe 2016, (8a))

'Lit. Mary remembered the year when John read Barriers, John Barriers.'

b. Mary-ka [John-i Barriers-lul ilk-ess-ki ttaymwuney nolla-ess-e, Mary-Nom John-Nom Barriers-Acc read because was.surprised John-i Barriers-lul.

John-Nom Barriers-Acc

(Abe 2016, (8b))

'Lit. Mary was surprised because John read Barriers, John Barriers.'

The repair by ellipsis approach makes the same prediction. In the contexts of $(5 a, b)$, however, two approaches diverge: while the in situ approach keeps the island effects intact, with no repair by ellipsis assumed, the repair by ellipsis approach cancels the island effects, thereby wrongly ruling in the examples.

\section{A Review of the Bi-clausal Approach to Gappy RDCs}

Abe (2016: 5) assumes that the RD element is an afterthought in a gappy RDC like (8a). Thus, its bi-clausal structure given in (8b) is paraphrased as (9), so that the process of adding afterthoughts is assumed to be captured.

(8) a. John-i e ilk-ess-e, Barriers-lul. John-Nom read-Past-Dec Barriers-Acc

'Lit. John read $e_{1}$, Barriers ${ }_{1}$ '

b. [TP John-i e ilk-ess-e], [TP Barriers-lul [TP John-i $t_{4}$ ilk-ess-eł] 
(9) $\exists x[$ John read $x] \&$ it was Barriers that John read

As I see, the interpretation of (9) is more likely to be associated with the example in (10), in which the gap in (8a) is overtly realized as an indefinite phrase mwuenka 'something' and the remnant in (8a) is in the focus position of the cleft clause (kukes-un = John-i ilkun-kes-un):

(10) John-i mwuenka-lul ilk-ess-e, kukes-un Barriers-i-ess-e.

John-Nom something-Acc read-Past-Dec it-Top Barriers-be-Past-Dec 'Lit. John read something, it was Barriers that John read.'

The indefinite phrase is then identified/specified as Barriers.

Accordingly, this raises a question as to whether the representation in (8b) correctly reflects the interpretation in (9). Unfortunately, if the gap in (8a) is filled with an indefinite phrase, which is supposed to be identified/specified by the RD element, the resulting gapless RDC is bad, as seen in (11a) (see also Ahn and Cho 2015: fn. 2); the source of (11a) is bad also, as seen in (11b).
(11) a. *John-i mwuenka-lul ilk-ess-e, Barriers-lul. John-Nom something-Acc read-Past-Dec Barriers-Acc 'Lit. John read something, Barriers.'
b. *John-i mwuenka-lul ilk-ess-e, John-i
John-Nom something-Acc read-Past-Dec John-Nom
Barriers-lul ilk-ess-e
Barriers-Acc read-Past-Dec
'Lit. John read something, John read Barriers.'

The accusative case marker on the $\mathrm{RD}$ element in (11a) suggests that it does not have a cleft source in (10). It is not derived by the in situ deletion as in (12a), either, due to the lack of strict identity in Abe's system. Thus it is to be obtained by Move \& Delete as in (12b).

(12) a. [TP John-i mwuenka-lul ilk-ess-e], [TP John-i Barriers-lul ilk-ess-e]

b. [TP John-i mwuenka-lul ilk-ess-e], [TP Barriers-lul [TP John-i t ilk-ess-ef] 
In (12b) the identity between the two clauses may be available at LF in which the indefinite phrase undergoes movement in the first clause:3)

(13) LF: [TP mwuenka-lul [TP John-i t ilk-ess-e]], [TP Barriers-lul [TP John-i t ilk-ess-e]]

Now if the derivation in (12b) is allowed under identity as in (13), the badness of (11a) remains to be accounted for.

On the other hand, if Abe assumes that the two clauses in (12b) are not strictly identical, (11a) cannot be derived, as desired. But then the gappy $\mathrm{RDC}$ in (8a) should be derived from the remaining cleft source in (10) in one of the following ways:

(14) a. [TP John-i

b. [TP John-i mwuenka-tul ilk-ess-e], [TP Barriers [TP kukes-un t-i-ess-e]]

In (14a), however, in situ deletion is not possible due to the lack of strict parallelism between the two clauses, as pointed out above. Further, after the deletion, it is hard to get the accusative case marker on the remnant. It is well known that the focused element in the cleft sentence does not bear the accusative case marker in Korean:

(15) John-i ilkun-kes-un/Kukes-un Barriers(*-lul)-iess-e.

In (14b), on the other hand, Move \& Delete has applied, and here parallelism requirement may be met in LF between the first clause and the cleft clause, as is often acknowledged in the name of syntactic accommodation (kukes-un $=$ John-i ilkun-kes-un):

(16) LF: [TP mwuenka-lul [TP John-i t ilk-ess-e]], [TP Barriers [TP John-i ilkun kes-un t-i-ess-e]]

3) Although Abe (2016) did not talk about LF parallelism, this is a possible extension for the data under consideration. 
Even if this is the case, however, the accusative case marker on the remnant is hard to get, as pointed out above. Besides, it is not entirely clear how the indefinite phrase in $(14 \mathrm{a}, \mathrm{b})$, not being identical to Barriers, can undergo backward deletion to derive the gappy RDC in (8a), repeated below.

(8) a. John-i e ilk-ess-e, $\quad$ Barriers-lul.
John-Nom read-Past-Dec Barriers-Acc
'Lit. John read $e_{1}$, Barriers $_{1}$ '.

A little better possibility is to apply backward deletion to the identical element, as illustrated below.4)

(17) a. [TP John-i Barriers-lul ilk-ess-e], [TP fohn-i Barriers-lul itk-ess-e],

b. [TP John-i Barriers-lul ilk-ess-e], [TP Barriers [TP John-i Barriers-lut ilk-ess-e]]

In a regular coordinate structure, however, backward deletion in fact produces a bad result:
(18) *John-i
Barriers-lul ilk-ess-e,
(kuliko) Bill-i
John-Nom Barriers-Acc read-Past-Dec and Bill-Nom
Barriers-lul ilk-ess-e
Barriers-Acc read-Past-Dec
'Lit. John read $e$, and Bill read Barriers.'

Thus it turns out that as seen in (17), backward deletion can be permitted only in the doubled coordinate structure.5) Put differently, the backward

4) In (17a) in situ deletion takes place in the second clause before backward deletion for reasons of strict parallelism. In (17b) Move \& Delete applies after backward deletion. I am not concerned with the choice between the two at the moment.

5) The backward deletion in question may apply to the same element in the adverbial clause in a complex sentence with some degradation:
(i) ??John-i Barriers-lut ilk-ki ceney, Bill-Nom Barriers-lul ilk-ess-e. John-Nom Barriers-Acc read before Bill-i Barriers-Acc read-Past-Dec
'Lit. Before John read e $e_{i}$, Bill read Barriers.'

My point, however, is that backward deletion does not apply to coordinate structures. 
deletion in (17) is a quite exceptional operation. This exceptionality can be eliminated if (8a) is not derived from the bi-clausal source, to which I will return at the end of this section.

One might say that there exists a null indefinite phrase corresponding to mwuenka 'something' in (10):
(19) John-i e ilk-ess-e, kukes-un Barriers-i-ess-e. John-Nom read-Past-Dec it-Top Barriers-be-Past-Dec 'Lit. John read e, it was Barriers that John read.'

But the fact that (11a), repeated below, is bad indicates that (8a) cannot involve this null indefinite phrase.

It is not entirely clear why the null indefinite phrase should be allowed in (8a), while its overt counterpart is not in (11a).

Alternatively, the gap in (8a) may be said to be a null definite pronoun that is coreferential with the RD element (cf. Takana 2001, Chung 2009). This means that the interpretation of (8a) is to be associated with the examples in (20), in which the gap in (8a) is realized as an overt pronoun, kukes 'it,' the source of (20a) being (20b).

(20) a. John-i kukes-ul ilk-ess-e, Barriers-lul i $_{\text {. }}$ John-Nom it-Acc read-Past-Dec Barriers-Acc

'Lit. John read it $t_{1}$, Barriers 1 .'

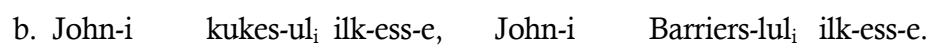
John-Nom it-Acc read-Past-Dec John-Nom Barriers-Acc read-Past-Dec 'Lit. John read $i t_{1}$, John read Barriers ${ }_{1}$ '

Under the bi-clausal analysis, this example will be derived as follows: 
(21) a. [John-i kukes-ul ilk-ess-e], [John-i Barriers-lul ${ }_{i}$ ilk-ess-e]

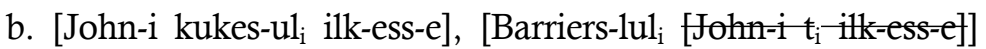

In (21a), the pronoun kukes, conveying old information, carries no focus in the first clause; its corresponding Barriers may not have a [Focus], either, contrary to the intention. Further, there is no strict identity between kukes and Barriers, not being a repetition of kukes, so the second clause will not undergo in situ deletion here. Then the remaining option is (21b). If the variable in the second clause in (21b), is non-distinct from the pronoun kukes in the first clause, Barriers-lul may serve as an $\mathrm{RD}$ element after deletion in favor of Abe's system.

To my intuition, however, (20a) as well as (20b) is bad (i.e., ?*). This is so because a pronoun is introduced in the first clause in the discourse while its antecedent appears later in the second clause here.6) This kind of cataphoric pronoun can hardly appear in the bi-clausal coordinate structure (juxtaposed by a covert conjunction, Chung 2009) as in (21).7) This point is strengthened by the multiple RDC in (22), which is worse than (20a).

6) The judgment for $(20 a, b)$ is shared by most of my informants. Two reviewers $(A, C)$, however, say that (20a) is acceptable, kekes being cataphoric to the $\mathrm{RD}$ element, Barriers. As I see, this kind of ameliorated cataphoric relation may result when this relation is pragmatically forced by the antecedent in the following conjunct. In a regular coordinate structure, in fact, backward pronominalization produces a bad result:

(i) *John-i ku kes-ul ilk-ess-e, (kuliko) Bill-i Barriers-lul ilk-ess-e John-Nom it-Acc read-Past-Dec and Bill-Nom Barriers-Acc read-Past-Dec 'Lit. John read $e$, and Bill read Barriers.'

Thus, for those speakers who allow (20a), backward pronominaliation is permitted only in the doubled coordinate structure in (20b), which sounds pretty awkward. This exceptionality can be eliminated if (20a) is not derived from the bi-clausal source in (20b). See also similar examples from English below from Heim (1982).

(ii) a. ${ }^{*} \mathrm{He}_{\mathrm{i}}$ came in, and a $\operatorname{man}_{\mathrm{i}}$ started yawning.

b. A $\operatorname{man}_{\mathrm{i}}$ came in, and he $\mathrm{i}_{\mathrm{i}}$ started yawning.

7) Note also that the following example (from fn. 5) cannot contain the overt cataphoric pronoun in place of the gap:

(i) *John-i kukes-ul $l_{\mathrm{i}}$ ilk-ki ceney, Bill-Nom Barriers-lul $\mathrm{i}_{\mathrm{i}}$ ilk-ess-e. John-Nom it-Acc read before Bill-i Barriers-Acc read-Past-Dec

'Lit. Before John read it $t_{\mathrm{i}}$, Bill read Barriers.' 
(22) ${ }^{*} \mathrm{Ku}-\mathrm{ka}_{1} \quad$ kukes-ul 2 ilk-ess-e, John-i $\mathrm{i}_{1}$ Barriers-lul $\mathrm{l}_{2}$ He-Nom it-Acc read-Past-Dec John-I Barriers-Acc 'Lit. $\mathrm{He}_{1}$ read it 2 , John 1 Barriers.'

Now that the overt pronoun kukes-ul is not allowed in (20a), its covert counterpart pro is also expected to be disallowed in (8a). In conclusion, the presumed gap in (8a) is neither an indefinite nor a definite pro. It would also be unreasonable to assume that pro has different properties from its overt counterpart here (see W Lee 2014 for relevant discussion).

Lastly, recall that under the bi-clausal analysis, the word order in (23a), repeated from (8a) without $e$, is derived from (23b), repeated from (8b).

(23) a. John-i ilk-ess-e Barriers-lul.

John-Nom read-Past-Dec Barriers-Acc 'John read Barriers.'

b. [TP John-i e ilk-ess-e], [TP Barriers-lul $1_{1}$ [TP John-i $t_{t}$ ilk-ess-e]]

In (23b), the first clause is repeated in the second clause, with Move $\&$ Delete applied. Thus, from the perspective of economy, a question arises as to why this clausal repetition is assumed at all if this clause is deleted again except the remnant. It appears that the repetition applies only to get the remnant Barriers-lul. It would be more economical if we can simply place Barriers-lul alone at the post-verbal position. Put differently, is the bi-clausal analysis correct? Together with the suspicious status of the gap in (23b), as discussed before, the answer seems to be in the negative.

In addition, I should like to point out that (23a) and (23b) are not really identical in their LF forms. In (23b), the lower TP-segment persists in LF although it is deleted in PF. To my intuition, however, in (23a) this TP is psychologically absent: it is never read silently. This means that (23b) is not a source for (23a).8)

In this situation, it is natural to turn to an alternative mono-clausal

8) In (23b) the remnant Barriers-lul is intended to carry a focus with it, but in (23a) it may not do so and it may also be replaced by the pronoun kukes-ul 'it-Acc' carrying an old information. 
source for (23a). ${ }^{9)}$ Its order can be derived by dislocating the object via rightward movement, as illustrated in (24a) (Ko 2016), or it can reflect the base head-initial order without positing the suspicious null category, as illustrated in (24b) (J-S Lee 2009, 2011a).

\section{a. John-i t ilk-ess-e Barriers-lul.

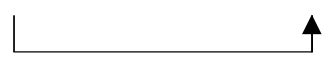 \\ b. John-i ilk-ess-e Barriers-lul. \\ (rightward movement) \\ (postverbal base object)}

The choice between the two will be made in section 7.10) Another question arises as to why gapless RDCs and gappy RDCs are derived differently in terms of non-constituent deletion and constituent deletion, respectively? I will attempt to eliminate this asymmetry in section 7 .

In short, the hybrid nature of Abe's (2016) in situ approach to gappy/gapless RDCs still requires a set of assumptions that need to be more justified. Below is a summary (more will be added as the discussion goes on):

- Head-final bi-clausal structure

- No repair by ellipsis

- Move \& Delete applies only to gappy RDCs

- In situ deletion applies only to gapless RDCs

- Non-constituent deletion (as well as constituent deletion) is allowed.

- Strict parallelism for deletion

9) For more arguments for the mono-clausal approach over the bi-clausal approach, see J-S Lee (2009, 2010b, 2011a, 2016a,b, 2017).

10) Ko $(2016,11)$ treats a gappy RDC like $(24 a)$ as a kind of cleft sentence (cf. also J-S Lee 2010a, 268 for a suggestion that a cleft sentence can be derived from a RDC like (24a)). She further claims that the RD element, called appendix, serves as a specificational argument that receives a focus or topic interpretation. But gappy RDCs may have different properties from cleft sentences (e.g., with regard to additive reading). An appendix, or the postverbal object, may also be interpreted as neither focus nor topic in some contexts, and the preverbal object is typically known to receive focus in Korean (cf. Kim 1985). 


\section{Intervention Effects}

Abe (2016: 7) extends his in situ deletion approach to the gapless multiple RD displaying intervention effects (attributed to Ko 2014 for Korean). Observe the following:

(25) a. *Mary-ka [John-i Barriers-lul ilk-ess-ta-ko] malha-ess-e, Mary-Nom John-Nom Barriers-Acc read-Past-Dec-Comp say-Past-Dec Mary-ka Barriers-lul.

Mary-Nom Barriers-Acc

'Lit. Mary said that John had read Barriers, Mary Barriers.'

b. ?Mary-ka [Barriers-lul John-i ilk-ess-ta-ko] malha-ess-e, Mary-Nom Barriers-Acc John-Nom read-Past-Dec-Comp say-Past-Dec Mary-ka Barriers-lul. Mary-Nom Barriers-Acc (Abe 2016: (9b))

'Lit. Mary said that Barriers John had read, Mary Barriers.'

The above contrast shows that adjacency must hold between the two $\mathrm{RD}$ elements in the original structure, which Abe calls intervention effects. In (25a) John- $i$ is an intervener between Mary-ka and Barriers-lul; in (25b), in which Barriers-lul is scrambled leftward to be adjacent to Mary-ka, there is no intervener between the two $\mathrm{RD}$ elements.

Abe (2016: 7) points out that the repair by ellipsis approach fails to capture the contrast in (25). This is because RD elements are obtained through movement in this approach, and thus (25a) is incorrectly allowed, as illustrated in (26a). (25b) is derived from (26b) without a wrong result.

(26) a. [TP Mary-ka [John-i Barriers-lul ilk-ess-ta-ko] malha-ess-e], [TP Mary-ka [TP Barriers-lul [TP <Mary-ka> John-i < Barriers-lul> itk-ess-ta-ko]] malha-ess-e]]]

(Abe 2016: (10a))

b. [TP Mary-ka [Barriers-lul John-i ilk-ess-ta-ko] malha-ess-e], [TP Mary-ka [TP Barriers-lul [TP < Mary-ka> [<Barriers-lul> John-i ilk-ess-ta-ko]] malha-ess-ef]]

(Abe 2016: (10b))

The in situ deletion approach is not able to handle the contrast in (25) directly, though. While (25b) can be derived correctly, as illustrated in (27b), (25a) would be wrongly allowed, as seen in (27a). 
(27) a. [TP Mary-ka [John-i Barriers-lul ilk-ess-ta-ko] malha-ess-e], [TP Mary-ka [John-i Barriers-lul ilk-ess-ta-ko] malha-ess-e]

b. [TP Mary-ka [Barriers-lul John-i ilk-ess-ta-ko] malha-ess-e], [TP Mary-ka [Barriers-lul John-i ilk-ess-ta-ko] malha-ess-e]]

(Abe 2016: $(11 a, b))$

Abe (2016: 8) thus resorts to the single remnant hypothesis to the effect that when more than one constituent is assigned a [Focus] feature in an ellipsis site, the constituents must form a single constituent by oblique movement (Takano 2002) which adjoins the lower constituent to the higher one. Thus (26b) is derived via oblique movement, as represented below.

(28) [TP Mary-ka [Barriers-lul John-i ilk-ess-ta-ko] malha-ess-e],

[TP [DP Mary-ka [DP Barriers-lul]] [<Barriers-lul $>$ John-i ilk-ess-ta-ko] [Focus] [Focus] malha-ess-e]

On the other hand, to exclude (27a), an additional condition on adjacency is required. Abe (2016) assumes that oblique movement is conditioned by the Minimize the Chain Links (henceforth, MCL), according to which adjunction of Barriers-lul to Mary-ka in (27a) cannot take place across the intervening argument, John-i, a possible landing site. One more condition on multiple $\mathrm{RD}$ elements is the c-command requirement: oblique movement applies only if the first $\mathrm{RD}$ element c-commands the second $\mathrm{RD}$ element. In (28) the c-command relation in question holds between the two RD elements: Mary-ka c-commands Barriers-lul. If this c-command relation is absent, it is predicted that multiple $\mathrm{RD}$ is not possible. This prediction is borne out, as illustrated below.

(29) *[Barriers-lul John-i ilk-ess-ta-ko] Mary-ka malha-ess-e, Barriers-Acc John-Nom read-Past-Dec-Comp Mary-Nom say-Past-Dec Barriers-lul Mary-ka. / Mary-ka Barriers-lul. Barriers-Acc Mary-Nom Mary-Nom Barriers-Acc (Abe 2016: (13)) 'Lit. That Barriers John had read Mary said, Barriers Mary / Mary Barriers.' 
The multiple $\mathrm{RD}$ elements cannot be derived in either order since Barriers-lul does not c-command Mary-ka or in the other way around.

In sum, to deal with intervention effects, Abe (2016) uses another set of assumptions:

- Single remnant hypothesis: multiple RDs must form a focus cluster together

- Focus clustering is achieved by oblique movement

- Oblique movement applies to arguments and is subject to MCL

- The first RD element must c-command the second $\mathrm{RD}$ element

\section{A Review of the Oblique Movement Approach}

A question arises as to why scrambling is bled by oblique movement, as shown in (26a), repeated below.

(26) a. [TP Mary-ka [John-i Barriers-lul ilk-ess-ta-ko] malha-ess-e], [TP Mary-ka [TP Barriers-lul [TP <Mary-ka> John-i <Barriers-lul $>$ ilk-ess-ta-ko]] malha-ess-e]]]

(Abe 2016: (10a))

If scrambling applies in the second clause, the two $\mathrm{RD}$ remnants will be wrongly attained. It is not obvious what could prevent the derivation in (26a). It appears that no known principles or conditions are violated here. Again, the question is why oblique movement takes precedence to scrambling. Other questions still remain. Why must multiple remnants form a single remnant constituent? Is oblique movement really motivated? It seems that all these questions largely remain to be answered.

Consider another type of multiple RDC containing an argumental remnant and a verbal complex remnant:

(30) a. Mary-ka [e Barriers-lul ilk-ess-ta-ko] malha-ess-e, Mary-Nom Barriers-Acc read-Past-Dec-Comp say-Past-Dec

Mary-ka ilk-ess-ta-ko.

Mary-Nom read-Past-Dec-Comp

'Lit. Mary said that she had read Barriers, Mary had read.' 
b. *Mary-ka [John-i Barriers-lul ilk-ess-ta-ko] malha-ess-e, Mary-Nom John-Nom Barriers-Acc read-Past-Dec-Comp say-Past-Dec Mary-ka ilk-ess-ta-ko.

Mary-Nom read-Past-Dec-Comp

'Lit. Mary said that John had read Barriers, Mary had read.'

Although the remnants are the same, (30a) contrasts with (30b). This contrast is obviously related to the presence of the null gap in (30a) and the absence of it in (30b). In (30a), the two remnants are apparently not adjacent. If the pre-ellipsis structure of the embedded clause in (30a) is like [Barriers-lul Mary-ka ilk-ess-ta-ko], they actually get adjacent. But the first remnant does not c-command the second one (assuming that the verbal complex is located in C), and the second remnant is not an argument. This is beyond the scope of Abe's system.

Next, consider the following example.

(31) a. Mary-ka [John-i congcong Barriers-lul ilk-ess-ta-ko] Mary-Nom John-Nom often Barriers-Acc read-Past-Dec-Comp malha-ess-e, Mary-ka John-i congcong. say-Past-Dec Mary-Nom John-Nom often 'Lit. Mary said that John often read Barriers, Mary John often.'

b. Mary-ka [John-i congcong Barriers-lul ilk-ess-ta-ko] Mary-Nom John-Nom often Barriers-Acc read-Past-Dec-Comp malha-ess-e, John-i congcong Barriers-lul. say-Past-Dec John-Nom often Barriers-Acc 'Lit. Mary said that John often read Barriers, John often Barriers.'

Among the three $\mathrm{RD}$ remnants, the adverb congcong 'often,' not being an argument, will not adjoin to the preceding argumental remnant within Abe's system. In (31b) Barriers-lul will not adjoin to the preceding adjunct congcong via oblique movement, either. Thus, the three remnants will not form a single remnant constituent. Or the single remnant hypothesis needs to be revised to the effect that adjuncts as well as arguments undergo oblique movement for focus clustering.

Finally, consider a RDC with non-argumental remnants given below.11)

11) The adverb congcong 'often' is inserted in the embedded clause to maintain the dele- 
(32) Mary-ka [John-i congcong Barriers-lul ilk-ess-ta-ko] malha-ess-e, Mary-Nom John-Nom often Barriers-Acc read-Past-Dec-Comp say-Past-Dec ilk-ess-ta-ko malha-ess-e. read-Past-Dec-Comp say-Past-Dec

'Lit. Mary said that John often had read Barriers, had read said.'

Above, the two verbal complexes, which are obviously non-arguments, serve as $\mathrm{RD}$ elements. The preceding verbal complex does not even c-command the following one. Again, this shows that Abe's system is not successful. In section 7, I will offer a different analysis that can avoid the problems with Abe's approach. The following is added to the sets of assumptions of Abe (2016).

- Oblique movement bleeds scrambling.

\section{Multiple Sluices, MCL with Equidistance}

Abe (2016: 9) finds further evidence for the in situ deletion approach from multiple sluices in Japanese. Consider the following examples from Korean that representatively illustrate his point.

(33) A: Nwukwunka-ka [nwukwunka-eykey ton-ul cwun] yeca-lul someone-Nom someone-Dat money-Acc gave woman-Acc chac-koiss-ess-ta.

look for-Prog-Past-Dec

'Lit. Someone was looking for a woman that gave someone money.'

B: Cengmal? Nwu-ka nwukwu-eykey?

Really? who-Nom who-Dat

'Really? Who to whom?'

tion process, otherwise all the arguments may be replaced by pros leaving the two remnants. 
(34) A: Nwukwunka-ka [John-eykey mwuenka-lul cwun] yeca-lul someone-Nom John-Dat something-Acc gave woman-Acc chac-koiss-ess-ta.

look for-Prog-Past-Dec

'Lit. Someone was looking for a woman that gave John something.'

B: Ung? *Nwu-ka mwues-ul?

Eh? who-Dat what-Acc

'Eh? Who what?'

Under Abe's oblique movement approach, the contrast between (33B) and (34B) can be handled. As shown below, the oblique movement in (35a) obeys MCL, but that in (35b) violates MCL, hence (33B) is good while (34B) is bad.

(35) a. [CP [TP [DP Nwu-ka [DP nwukwu-eykey]] [<nwukwu-eykey> ton-ul ewun] [Focus] [Focus] yeea-tul chac-koiss-ess-ni] Q]

b. [CP [TP [DP Nwu-ka [DP mwues-ul]] fohn-eykey <meeka>-ul ewur] [Focus] [Focus] yeea-lul chac-koiss-ess-ni] Q]

Abe $(2016,13)$ also discusses a case where oblique movement apparently takes place between the two non-adjacent remnants:

(36) A: Nwukwunka-ka Mary-eykey mwuenka-lul cwu-ess-ta. someone-Nom Mary-Dat something-Acc gave-Past-Dec 'Someone gave Mary something.'

B: Ung? Nwu-ka mwues-ul?

Eh? who-Dat what-Acc

'Eh? Who what?'

It appears that mwues-ul 'what-Acc' crosses Mary-eykey 'Mary-Dat' to adjoin to nwu-ka 'who-Nom,' violating MCL. Abe $(2016,13)$ avoids this problem by using the notion of equidistance (Chomsky 1993): under the assumption that V-to-v movement makes VP and vP the same maximal domain for measuring equidistance, nwu-ka and Mary-eykey are equidistant to mwrues-ul within vP, hence the oblique movement of mwres-ul to nwr-ka 
over Mary-eykey does not violate MCL. The same treatment can extend to a gapless multiple RDC like (37).

(37) Mary-ka John-eykey chayk-ul cwu-ess-ta, Mary-ka chayk-ul. Mary-Nom John-Dat book-Acc gave-Past-Dec Mary-Nom book-Acc 'Lit. Mary gave John a book, Mary a book.' (Abe 2016, (24))

Here, the apparent violation of MCL with the oblique movement of chayk-ul to Mary-ka across John-eykey can be evaded in terms of the notion equidistance.

Again, there are examples that are recalcitrant to Abe's oblique movement analysis.

(38) A: Nwukwunka-ka Mary-lul etinka-lo teylyega-ess-ta. someone-Nom Mary-Acc somewhere-to take-Past-Dec 'Someone took Mary somewhere.'

B: Ung? Nwu-ka eti-lo?

Eh? who-Dat where-to

'Eh? Who where?'

(39) A: John-i nwukwunka-lul etinka-lo teylyega-ess-ta. John-Nom someone-Acc somewhere-to take-Past-Dec 'John took someone somewhere.'

B: Ung? Nwukwu-lul eti-lo?

Eh? who-Acc where-to

'Eh? Who where?'

First of all, in the above examples the sluice remnant eti-lo 'where' is not an argument but an adjunct. But the fact here suggests that this adjunct undergo oblique movement (recall also (31)). The following is a relevant structure in which the adjunct eti is adjoined to vP:

(40) [TP Subj-ka [vP [vp Subj-ka [VP Obj-lul V] V-v] eti] V-v-T] 
In (38), however, it is not adjacent to the subject due to the object intervener, although it is c-commanded by the subject in Spec TP; in (39), it is not c-commanded by the object. Thus there is no way for the adjunct in question to undergo oblique movement in Abe's system.

Therefore, a different analysis is called for. I will attempt to offer one in the next section. Finally, the following is added to the sets of assumptions Abe (2016) adopts.

- MCL is relaxed in the equidistant domain.

\section{A Head-initial Approach: Mono-clausal Source, TP-raising}

Now it is time to choose between (24a) and (24b), repeated below.
a. John-i t ilk-ess-e Barriers-lul.
(rightward movement)
b. John-i ilk-ess-e Barriers-lul.
(postverbal base object)

In fact, I have argued for the head-initial mono-clausal approach to gappy RDCs in Korean and argued against the rightward movement approach to them (J-S Lee 2009, 2011a, 2016b). To show that (24b) is over (24a), I introduce just one piece of empirical evidence in the area of binding for space reasons (see also J-S Lee 2009, 2011a, 2016b for more discussion). Consider:

(41) a. Selo 1 -uy sensayngnim-i [John-kwa Mary $]_{1}-$ lul pinanha-ess-ta. each other-Gen teacher-Nom John-and Mary-Acc criticize-Past-Dec 'Each other's teacher criticized John and Mary.'

b. [John-kwa Mary] ${ }_{1}-$ lul selo ${ }_{1}$-uy sensayngnim-i $t_{1}$ pinanha-ess-ta. John-and Mary-Acc each other-Gen teacher-Nom criticize-Past-Dec 'John and Mary each other's teacher criticized.'

c. *Selo-uy sensayngnim-i $t_{1}$ pinanha-ess-ta [John-kwa Mary] ${ }_{1}-1 \mathrm{lul}$. each other-Gen teacher-Nom criticize-Past-Dec John-and Mary-Acc 'Each other's teacher criticized John and Mary.' 
Since the rightward movement approach assumes a head-final structure in Ko (2016), for instance, (41b) is derived from the base (41a) by leftward movement of the object, [John and Mary]. Importantly, this movement creates a new binding relation. With this in mind, let us take a look at (41c). This example is derived from the base (41a) by rightward movement of the object, [John and Mary]. As the object is now structurally higher than the subject containing the anaphor, just as in (41b), it should be able to bind the anaphor within the subject. Unlike (41b), however, (41c) does not allow new binding relation. This state of affairs tells us that the rightward movement approach is not on the right track. ${ }^{12)}$ Notice also that the bi-clausal approach cannot account for the ungrammaticality of (41c), either: the RD element should be able to bind the anaphor in the second clause just as in (41b), contrary to fact (see J-S Lee 2016: 2.3.1, 4.3.8 for more discussion). ${ }^{13)}$

12) It might be said that in (41c) the object undergoes rightward movement to a position structurally lower than the subject (this requires the lexicalist hypothesis in Korean). This conjecture, however, does not hold in that when a complex sentence is considered, the same contrast emerges:

(i) a. [John-kwa Mary] $]_{1}$ lul [na-nun [selo1-uy sensayngnim-i $t_{1}$ John-and Mary-Acc I-Top each other-Gen teacher-Nom pinanha-ess-ta-ko] mitnunta]]. criticize-Past-Dec-Comp believe 'Lit. John and Mary, I believe that each other's teacher criticized t.'

b. *[na-nun [selo-uy sensayngnim-i $t_{1}$ pinanha-ess-ta-ko] I-Top each other-Gen teacher-Nom criticize-Past-Dec-Comp mitnunta]] [John-kwa Mary] $1-1$ lul. believe John-and Mary-Acc

'Lit. I believe that each other's teacher criticized t, John and Mary.'

A reviewer (B) claims that the rightward movement approach can still be sustainable if we assume that the rightward movement is restricted by Ross's Right Roof Constraint, which would block the rightward movement of the antecedent out of the embedded clause in (ib). But the contrast between (ia) and (ib) disappears if the anaphor selo-uy 'each other-Gen' is eliminated, which means that the movement in question is actually possible. And the badness of (41c) remains to be accounted for. Although I do not agree, another reviewer (C) finds (41c) and (ib) good.

13) A reviewer (B) also claims that the ellipsis approach could account for (41c) since nothing binds the subject reflexive in the first clause (with the assumption that pro does not move), as shown in (i).

(i) * Selo $_{1}$-uy sensayngnim-i pro ${ }_{1}$ pinanha-ess-ta, [John-kwa Mary $]_{1}$-lul. each other-Gen teacher-Nom criticize-Past-Dec John-and Mary-Acc 
Under the head-initial mono-clausal approach, the contrast in (41) can be easily accounted for. Consider:

(42) a. Selo 1 -uy sensayngnim-i pinanha-ess-ta [John-kwa Mary $]_{1}-1$ lul. each other-Gen teacher-Nom criticize-Past-Dec John-and Mary-Acc 'Each other's teacher criticized John and Mary.'

b. [John-kwa Mary $]_{1}-$ lul selo ${ }_{1}$-uy sensayngnim-i pinanha-ess-ta $t_{1}$. John-and Mary-Acc each other-Gen teacher-Nom criticize-Past-Dec 'John and Mary, each other's teacher criticized.'

c. *Selo 1 -uy sensayngnim-i pinanha-ess-ta [John-kwa Mary $]_{1}-$ lul. each other-Gen teacher-Nom criticize-Past-Dec John-and Mary-Acc 'Each other's teacher criticized John and Mary.'

(42b) is derived from the base (42a) by leftward movement of the object, [John and Mary]. As expected, this movement creates a new binding relation. (42c) reflects the base (42a), with no object shift having taken place over the verbal complex. As the object is thereby structurally lower than the subject containing the anaphor, it is unable to bind the anaphor within the subject, as desired.

Under the head-initial mono-clausal approach, a gapless RDC like (43) can be derived, as illustrated in (44).

(43) John-i Barriers-lul ilk-ess-e Barriers-lul. John-Nom Barriers-Acc read-Past-Dec Barriers-Acc 'Lit. John read Barriers, Barriers.'

(44) John-i Barriers-lul ilk-ess-e Barriers-lul.

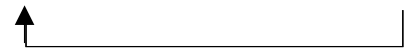

This approach, however, cannot deal with the following example.

(ii) a. ${ }^{*}$ Na-nun pro $_{\mathrm{i}}$ caki-uy $y_{\mathrm{i}}$ sensayngnim-eykey chwuchenha-ess-ta, Yuni-lul ${ }_{\mathrm{i}}$. I-Top self-Gen teacher-Dat recommend-Past-Dec Yuni-Acc 'Lit. I recommended to self's teacher, Yuni.'

b. Na-nun Yuni-lul $l_{i}$ caki-uy ${ }_{i}$ sensayngnim-eykey chwuchenha-ess-ta.

'I recommended Yuni to self's teacher.'

In (iia) pro can bind the anaphor in the first clause (see (iib) for why), but the result is still bad. 
The copy left behind by Object Shift remains undeleted, or phonetically recovered. I assume that this copy results from copy reflexivization in the extended sense of Hornstein (2001). That is, the RD copy is treated as a kind of anaphor, and this anaphor is used for emphasis or confirmation. 14)

Now the next task is how to derive a gapless multiple RDC like (1), repeated below.
(1) John-i
Barriers-lul ilk-ess-e, John-i
Barriers-lul.
John-Nom Barriers-Acc read-Past-Dec John-Nom Barriers-Acc 'Lit. John read Barriers, John Barriers.' (Abe 2016, (1))

If the above type of gapless RDC in (1) is to be derived under the mono-clausal approach, it may be derived with more derivational steps, as illustrated below. 15 )

(45)
a. John-i ilk-ess-e Barriers-lul
$=>$ Object Shift
b. John-i [Barriers-lul [ilk-ess-e]] => Move verb
c. [ilk-ess-e [John-i [Barriers-lul]]] $=>$ Move Subj, Object
d. [John-i [Barriers-lul [ilk-ess-e [<John-i $>[<$ Barriers-lul $>$ ]]]]]

In (45d), the traces left behind are phonetically recovered for emphasis or confirmation, producing the surface in (1). Another possible derivation is conceivable:

14) In Hornstein (2001), the object John in (i) is left behind by A-movement of it to the subject position, and it is analyzed as a reflexive copy. This analysis may apply to the Korean example in (ii), also.

(i) John admires John $=>$ himself

(ii) John-un John-ul chwuchenha-ess-ta.

John-Top John-Acc recommend-Past-Dec

'John recommended himself.'

In this connection, the post-verbal object copy left behind by A-movement of it to the pre-verbal object position in (44) may also be treated as a reflexive anaphor.

15) Under the head-initial structure, the verbal complex is formed by leftward head movement of the verb to the relevant functional categories, and the resulting complex can further move to a higher functional position, as seen in (45c). For details about the head-initial structure, see J-S Lee (2009, 2010b, 2011b). 
(46) a. John-i ilk-ess-e Barriers-lul => Object Shift

b. John-i [Barriers-lul [ilk-ess-e]] => Move Subj, Object

c. John-i [Barriers-lul [TP $<$ John-i $>[<$ Barriers-lul $>$ [ilk-ess-e] $]]]=>$ TP-raising

d. $[$ TP $<$ John-i $>$ [<Barriers-lul $>$ [ilk-ess-e]]] [John-i [Barriers-lul ...]] $(=>(1))$

In (46d), the traces left within the raised TP are phonetically recovered for emphasis or confirmation, producing the surface in (1). ${ }^{16)}$

This approach, however, faces difficulties in dealing with the contrast associated with intervention effects, repeated below.

(25) a. *Mary-ka [John-i Barriers-lul ilk-ess-ta-ko] malha-ess-e, Mary-Nom John-Nom Barriers-Acc read-Past-Dec-Comp say-Past-Dec

Mary-ka Barriers-lul.

Mary-Nom Barriers-Acc

(Abe 2016: (9a))

'Lit. Mary said that John had read Barriers, Mary Barriers.'

b. ?Mary-ka [Barriers-lul John-i ilk-ess-ta-ko] malha-ess-e,

Mary-Nom Barriers-Acc John-Nom read-Past-Dec-Comp say-Past-Dec

Mary-ka Barriers-lul.

Mary-Nom Barriers-Acc

(Abe 2016: (9b))

'Lit. Mary said that Barriers John had read, Mary Barriers.'

Both (25a) and (25b) will be derived in the equal manner: Mary-ka and Barriers-lul will front out of the embedded clause, and then the remaining clause will front again to derive their surfaces, with the trace positions overtly filled by phonetic recovering in the manner illustrated in (46) (not in the manner illustrated in (45)). Hence, the above contrast remains unaccounted for. The adjacency between the two remnants in the original given order must be respected in one way or another.

Under the mono-clausal approach, I propose an alternative analysis to gapless multiple RDCs. As for (1), I suggest the following derivation:

16) Under the above approach, (11a) can be ruled out by theta theory; (20a) is derived by backward pronominalization of the higher object copy in (44), and the resulting badness can be ascribed to Condition C violation; (22) is derived by the impossible backward pronominalization of the raised Subject and Object in (45d) or in (46d). 
(47) a. John-i ilk-ess-e Barriers-lul => Object Shift

b. [TP John-i [Barriers-lul [ilk-ess-e]]] $=>$ TP-raising to Spec CP

c. [CP [TP John-i [Barriers-lul [ilk-ess-e]]] C [TP-John-i [Barriers-lul [ilk-ess-e]\}] => phonetic resumption of Subj and Obj

d. [CP [TP John-i [Barriers-lul [ilk-ess-e]]] C $[\mathrm{TP}<$ John-i $>[<$ Barriers-lul $>]]=>(1)$

In (47b) TP-raising to Spec CP applies to produce the TP doubling effects, but the resulting structure is different from the previous bi-clausal coordinate structure. This TP-raising would not apply if it is not motivated by the presence of $\mathrm{RD}$ elements. I assume that in (47c) the strikethroughed clausal TP-copy is further open to phonetic recovering before it is finally unpronounced. In (47d), two remnant copies within the lower TP-copy are phonetically recovered for the purpose of emphasis or confirmation. I call this process phonetic resumption (see also J-S Lee 2010c). Accordingly, the gapless multiple RDC in (1) is derived. Here the crucial assumption is that a deleted copy can undergo phonetic resumption for emphasis or confirmation before it eventually becomes mute.17)

To deal with the remaining intervention effects, then, the adjacency relation between the multiple remnants needs to be incorporated into the phonetic resumption of the remnants in the current system. For $(24 a, b)$, only relevant parts are presented in $(48 \mathrm{a}, \mathrm{b})$ to implement this factor:

(48) a. ${ }^{*}<$ Mary-ka> †ohn-i <Barriers-lul $>$ itk-ess-ta-ko\} malha-ess-e, b. ?<Mary-ka $>$ [<Barriers-lul $>$ John-i illk-ess-ta-ko] malha-ess-e,

The above contrast suggests that the phonetic resumption of the $\mathrm{RD}$ remnants requires adjacency - in (48a) the adjacency between the two remnants is absent but in (48b) they are adjacent. The process of phonetic resumption here can be regarded as a partial linearization of sounds (of words) from left to right in PF. (Note that the current head-initial structure is optimized for linearization (Kayne 1994).) In this sense, it can be said that phonetic linearization has to proceed in a continuous sound flow. This conjecture is corroborated by the following view of the contrast be-

17) A similar case is a resumptive pronoun. An island violation is neutralized when a trace in the island is phonetically recovered with a resumptive pronoun (see Hornstein, Lasnik and Uriagereka 2003 for relevant discussion). 
tween (48a) and (48b). In (48b), there is no pause between the two RD remnants as they are adjacent, so that the sound flow on them can be continuous. In (48a), by contrast, the sound flow in question is discontinuous owing to the intervener in between, namely, John-i, which may be called a potential remnant. The adjacency requirement under concern may then be attributed to a PF principle like (49), which I propose below:

(49) Phonetic resumption must proceed in a continuous stream of sound.

It follows then that phonetic resumption applies only to the adjacent materials with no intervening potential remnant. The ungrammaticality of (29) also follows without further explanation.

In passing, observe that in (47d), a case marker can be further deleted on the final remnant but not on the first remnant, as seen below:

(50) a. [John-i Barriers-lul ilk-ess-e] [<John-i $><$ Barriers-lul $>$ ilk-ess-e]

b. *[John-i Barriers-lul ilk-ess-e] [<John-i $><$ Barriers-lul $>$ ilk-ess-e $]$

An (2016) deals with the above contrast in terms of Extra Deletion which deletes the case particle only on the final remnant at PF. Under the current approach, phonetic resumption may apply to exclude the case marker only on the final remnant at $\mathrm{PF}$ since it requires adjacent materials with no deleted material in between to maintain a continuous sound flow. Then the Extra Deletion effects may fall out.

Notice also that this approach shares fundamentally the same spirit as the oblique movement approach coupled with MCL under the single remnant hypothesis in Abe (2016). But the current approach has a wider range of coverage. To see this, reconsider (31a), repeated below.

(31) a. Mary-ka [John-i congcong Barriers-lul ilk-ess-ta-ko] Mary-Nom John-Nom often Barriers-Acc read-Past-Dec-Comp malha-ess-e, Mary-ka John-i congcong. say-Past-Dec Mary-Nom John-Nom often 
'Lit. Mary said that John often read Barriers, Mary John often.' Three consecutive remnants can be phonetically recovered since they are adjacent to one another regardless of their status as argument or adjunct, as seen in (51) (the same point applies to (31b) as well):

(51) $<$ Mary-ka $>$ [<John-i > < congcong > Barriers-lul ilk-ess-ta-ko]

Island insensitivity of multiple gapless RDCs can be handled with no difficulty. One relevant example is repeated from (7a) beow.

(7) a. Mary-ka [John-i Barriers-lul ilk-un] hay-lul kiekha-ess-e, Mary-Nom John-Nom Barriers-Acc read year-Acc remembered, John-i Barriers-lul.

John-Nom Barriers-Acc

(Abe 2016, (8a))

'Lit. Mary remembered the year when John read Barriers, John Barriers.'

Under the current phonetic resumption approach, (7a) is derived as illustrated below. Only the most relevant steps are offered:

(52) a. [CP [TP Mary-ka [John-i Barriers-lul ilk-un] hay-lul kiekha-ess-e] C [TP Mary-ka [John-i Barriers-lul ilk-un] hay-lul kiekha-ess-e]]] $=>$ phonetic resumption of Subj and Obj in the embedded clause

b. [CP [TP Mary-ka [John-i Barriers-lul ilk-un] hay-lul kiekha-ess-e] C [TP Mary-ka [<John-i> <Barriers-lul> ilk-un] hay-lul kiekha-ess-e]t] $=>(7 a)$

In (52a) TP-raising has applied, and within the to be unpronounced TP-copy, two consecutive remnants in the embedded clause are phonetically recovered for emphasis or confirmation, resulting in the surface (7a). Here the absence of island effects are observed simply because no movement has taken place out of the island.

If a movement takes place out of an island, island effects are expected to appear, as borne out in (5a), for instance, repeated below. 
(5) a. ?*Mary-ka [John-i e ilk-un] hay-lul kiekha-ess-e,

Mary-Nom John-Nom read year-Acc remembered,

Barriers-lul.

Barriers-Acc

(Abe 2016, (4a))

'Lit. Mary remembered the year when John read $e_{1}$, Barriers ${ }_{1}$.'

Under the current approach, (5a) is derived as illustrated with the most relevant steps below.

(53) a. [TP Mary-ka [John-i Barriers-lul ilk-un] hay-lul kiekha-ess-e]] $=>$ Move Barriers-lul out of the island

b. [TP Barriers-lul [TP Mary-ka [John-i e ilk-un] hay-lul kiekha-ess-e]]] $=>$ Lower TP-raising to Spec CP

c. [CP [TP Mary-ka [John-i e ilk-un] hay-lul kiekha-ess-e] C [TP Barriers-lul [TP Mary-ka [John-i e ilk-un] hay-lul kiekha-ess-e]] $=>(5 a)$

In (53b) Barriers-lul has moved out of the island, and the resulting island effects persist through the derivation, with no repair. TP-copy deletion in $(53 c)$ is of no help since island violation configuration is still left in the raised TP: the phonetic gap in the island causes island effects (for related discussions, see J-S Lee 2010c, attributed to Hornstein, Lasnik and Uriagereka 2003, according to which island effects do not result from movement, and movement from islands is permitted just in case a phonetically null trace does not result).

This approach must also be able to derive the following gapless multiple $\mathrm{RDC}$, repeated from (37).

(37) Mary-ka John-eykey chayk-ul cwu-ess-ta, Mary-ka chayk-ul. Mary-Nom John-Dat book-Acc gave-Past-Dec Mary-Nom book-Acc 'Lit. Mary gave John a book, Mary a book.'

(Abe 2016, (24))

The two RD remnants are not adjacent on the surface, so Abe (2016) resorts to the notion of equidistance in applying the oblique movement to evade the MCL violation. Under the head-initial hypothesis, the initial clause will be derived in the following steps: 
(54) a. [TP Mary-ka cwu-ess-ta John-eykey chayk-ul]

b. [TP Mary-ka chayk-ul cwu-ess-ta John-eykey <chayk-ul>]

c. [TP Mary-ka John-eykey chayk-ul cwu-ess-ta <John-eykey $><$ chayk-ul $>$ ]

I first assume that within $\mathrm{vP}$ in (54c), the preverbal indirect object and the direct object are equidistant to the subject, as Abe (2016) also does. In this connection, I propose that after TP-raising to Spec CP in (53c), its TP-copy can be reconstructed as (54b), in which the indirect object has returned to its original postverbal position and thereby Mary-ka and chayk-ul have become adjacent. As a result, the $\mathrm{RD}$ remnants in (37) finally obtain. ${ }^{18)}$ Crucially, the reconstruction in question is possible through a previous record which is accessible only under the head-initial structure I am adopting.

This treatment can further extend to deal with the contrast, repeated below.

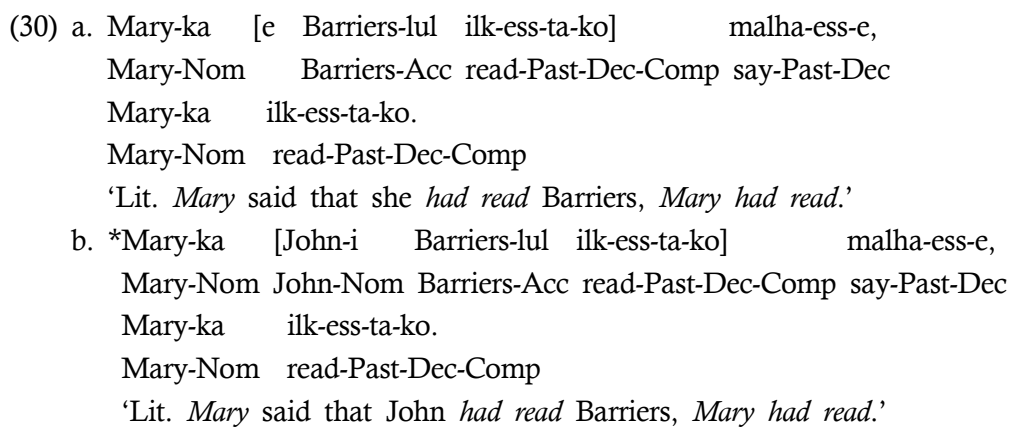

In (30b) Mary-ka is not adjacent to ilk-ess-ta-ko, hence (30b) is not possible. By contrast, (30a) is possible. Obviously, this is due to the presence of the null category coreferential with the matrix subject. If this null category results from deletion or null pronominalization of Mary-ka, it can be assumed to be phonetically recovered, so that it may physically appear as a remnant. This possibility also accounts for a gapless multiple RDC like (55), given below.

18) In (37), Mary-ka and cwu-ess-ta 'gave' can also appear as multiple RD remnants. Thus, reconstruction to (54a) seems possible as well. But this pair of multiple RD remnants may also appear as a result of VP-ellipsis or complement deletion. 
(55) Mary-ka [e Barriers-lul ilk-ess-ta-ko] malha-ess-e, Mary-Nom Barriers-Acc read-Past-Dec-Comp say-Past-Dec Mary-ka Barriers-lul.

Mary-Nom Barriers-Acc

'Lit. Mary said that she had read Barriers, Mary Barriers.'

Going back to (30a), a question arises as to how Mary-ka gets adjacent to the verbal complex ilk-ess-ta-ko. As in Abe (2016), the notion equidistance may be invoked here as well; that is, within vP the subject and the object are in the equi-distant domain of the verb. But the verbal complex cannot undergo oblique movement as it is not an argument. Under the head-initial structure, however, the embedded clause of (30a) can have the following derivational steps, as roughly suggested above.

(56) a. [Mary-ka ilk-ess-ta Barriers-lul] $=>$ Object Shift, Subject Raising

b. [TP Mary-ka [Barriers-lul <Mary-ka> ilk-ess-ta]] $=>$ TP-raising to CP

c. [TP Mary-ka Barriers-lul <Mary-ka > <ilk-ess-ta]-ko>

If (56c) is taken via reconstruction, the string [Mary-ka ilk-ess-ta-ko] can be phonetically resurrected, as desired. Now (32) is easy to derive under the current approach as the two remnants are adjacent.

(32) Mary-ka [John-i congcong Barriers-lul ilk-ess-ta-ko] malha-ess-e, Mary-Nom John-Nom often Barriers-Acc read-Past-Dec-Comp say-Past-Dec ilk-ess-ta-ko malha-ess-e. read-Past-Dec-Comp say-Past-Dec 'Lit. Mary said that John often had read Barriers, had read said.'

Lastly, let us consider the following example.

(57) Mary-ka [e Barriers-lul ilk-ess-ta-ko] malha-ess-e, Mary-Nom Barriers-Acc read-Past-Dec-Comp say-Past-Dec Barriers-lul Mary-ka.

Barriers-Acc Mary-Nom

'Lit. Mary said that she had read Barriers, Barriers Mary.' 
The surface order in the embedded clause in (56) is the same as that in (57a), in which it is possible that the null category is phonetically recovered as Mary-ka as in (55b).

(57) a. [Barriers-lul e ilk-ess-ta-ko]

b. [Barriers-lul <Mary-ka> ilk-ess-ta-ko]

As a result, the remnants in the reversed order in (56) can be derived.

Now the remaining discussion is how to deal with the contrast in multiple fragments observed previously, repeated below.

(33) A: Nwukwunka-ka [nwukwunka-eykey ton-ul cwun] yeca-lul someone-Nom someone-Dat money-Acc gave woman-Acc chac-koiss-ess-ta.

look for-Prog-Past-Dec

'Lit. Someone was looking for a woman that gave someone money.'

B: Cengmal? Nwu-ka nwukwu-eykey?

Really? who-Nom who-Dat

'Really? Who to whom?'

(34) A: Nwukwunka-ka [John-eykey mwuenka-lul cwun] yeca-lul someone-Nom John-Dat something-Acc gave woman-Acc chac-koiss-ess-ta.

look for-Prog-Past-Dec

'Lit. Someone was looking for a woman that gave John something.'

B: Ung? *Nwu-ka mwues-ul?

Eh? who-Dat what-Acc

'Eh? Who what?'

Since fragments are not part of the previous sentence, it is not clear how the current TP-raising analysis will apply to get the multiple sluices. I first observe that as a reply to (33A), for instance, the following full $\mathrm{RDC}$-like answer is also possible: 
(33) B': Cengmal? Nwu-ka [nwukwu-eykey ton-ul cwun] yeca-lul Really? who-Nom who-Dat money-Acc gave woman-Acc chac-koiss-ess-ni, nwu-ka nwukwu-eykey? look for-Prog-Past-Q who-Nom who-Dat

'Lit. Who was looking for a woman that gave whom money, who whom?'

This fact suggests that multiple fragments in (33B) can be derived from (33B') through the ellipsis of the preceding full clause (cf. Park and Shin 2016):19)

(58) Cengmal? [TP Nwu-ka [nwukwu-eykey ton-ul ewun] yeea-hut chac-koiss-ess-ni\}, nwu-ka nwukwu-eykey?

This opens the way for TP-raising, followed by the phonetic resumption of the remnants. This possibility is illustrated below. ${ }^{20)}$

(59)

$$
\begin{aligned}
& {\left[\begin{array}{lll}
\mathrm{CP} & {[\mathrm{TP}} & \ldots . . . .
\end{array}\right] \mathrm{C}[\mathrm{TP}<\mathrm{Nwu}-\mathrm{ka}>\quad[<\text { nwukwu-eykey }>\text { ton-ut }} \\
& \text { ewun] yeea-tul chac-koiss-ess-nif] }]=>(33 \mathrm{~B})
\end{aligned}
$$

On the other hand, the two remnants are not adjacent in (34), hence phonetic resumption cannot occur.

Other types of RDCs, not discussed Abe (2016) and in this paper, are outside the scope of this paper, for example, mixed RDCs like (60a) (see,

19) Park and Shin (2016: 56) propose essentially the same analysis: the fragment answer right after the polarity answer particle such as ung 'yes' or ani 'no' in (iA) is actually a $\mathrm{RD}$ element, as analyzed in (iA').

(i) Q: Chelswu-ka mek-ess-ni?

Chelswu-Nom eat-Past-Q

'Did Chelswu eat?'

A: Ani, amwukes-to.

No anything

'No, anything.'

A': Ani, [TP Chelswu-ka e mek-ess-e], amwukes-to.

No Chelswu-Nom eat-Past-Dec anything

(their (6Q))

(their (6A))

(their (6'A))

20) Out of the preceding deleted TP-copy can one or two wh-phrases be recovered, for example, Nwu-ka, nwu-ka nwukwu-eykey?, or Nwukwu-eykey, nwu-ka nwukwu-eykey? These examples are not derived by other approaches (e.g., Abe's 2016 in situ approach, Chung 2009 type Move \& Delete approach). 
e.g., Park and Kim 2016) and split RDCs like (60b) (see, e.g., Ko 2016):

(60) a. Mary-ka [John-i e ilk-ess-ta-ko] malha-ess-e, Mary-Nom John-Nom read-Past-Dec-Comp say-Past-Dec Barriers-lul Mary-ka. / Barriers-lul John-i. Barriers-Acc Mary-Nom Barriers-Acc John-Nom 'Lit. Mary said that John had read e, Barriers Mary/Barriers John.' b. Mary-ka [John-i e seypen ilk-un] hay-lul kiekha-ess-e, Mary-Nom John-Nom three times read year-Acc remembered, Barriers-lul.

Barriers-Acc

'Lit. Mary remembered the year when John read $e$ three times, Barriers.'

I merely say that the current analysis can possibly accommodate these types of RDCs. For the derivation of (60a), movement of Barriers-lul, TP-raising, and phonetic resumption of Mary-ka/John-i can apply (see J-S Lee 2017 for a different analysis). For (60b), movement of Barriers-lul yields an island violation, but the island effects here can be neutralized by pseudo-resumption of the frequency element seypen in that this element may compensate the phonetic gap created by the movement of Barriers-lul (see J-S Lee 2010c for relevant discussion). I leave this topic for another study.

\section{Summary and Conclusion}

To derive multiple $\mathrm{RD}$ elements and fragments, we have seen that Abe's (2016) in situ approach needs the following assumptions collected from the discussions so far made:

- Head-final bi-clausal structure

- No repair by ellipsis

- Move \& Delete applies only to gappy RDCs

- In situ deletion applies only to gapless RDCs

- Non-constituent deletion (as well as constituent deletion) is allowed.

- Strict parallelism for deletion 
- Single remnant hypothesis: multiple RDs must form a focus cluster together

- Focus clustering is achieved by oblique movement

- Oblique movement applies to adjacent arguments and is subject to MCL

- Oblique movement bleeds scrambling.

- The first $\mathrm{RD}$ element must c-command the second $\mathrm{RD}$ element

- MCL is relaxed in the equidistant domain

Some of the assumptions remain stipulative and limited, and they even produce wrong results. On the other hand, the current approach needs the following:

- Head-initial mono-clausal structure

- TP-raising

- Phonetic resumption that requires adjacency.

It is seen that the current approach is much simpler than Abe's (and arguably other similar analyses) and has a wider range of coverage than his. The issues of non-constituent deletion, oblique movement, focus clustering, and the like never arise. Under the mono-clausal analysis, island effects appear in gappy RDCs simply as a result of a movement out of an island leaving a phonetic gap in the island. Crucially, phonetic resumption is proposed to derive gapless multiple $\mathrm{RD}$ remnants and fragments in terms of TP-raising. The proposed analysis benefitted from the head-initial hypothesis. Some effects such as island repair effects, intervention effects and Extra Deletion effects are also seen to follow from the current approach.

\section{References}

Abe, Jun. (2016). Against the "repair by ellipsis" approach: The case of Japanese multiple right dislocation. In Tae Sik Kim and Seungwan Ha, eds., Proceedings of the 18th Seoul International Conference on Generative Grammar. Beyond core syn- 
tax: A minimalist approach, 3-16. Sogang University.

Ahn, Hee-Don and Sungeun Cho. (2015). Right dislocation vs. fragment: A reply to Ko (2014). Studies in Generative Grammar 25, 427-446.

An, Duk-Ho. (2016). Extra deletion in fragment answers and its implications. Journal of East Asian Linguistics 25.4, 313-350.

Chomsky, Noam. (1993). A minimalist program for linguistic theory. In Ken Hale and Sammuel J. Keyser, eds., The view from building 20: Essays in linguistics in honor of Sylvian Bromberger, 1-52. Cambridge, MA: MIT Press.

Chung, Daeho. (2009). An elliptical coordination analysis of the right dislocated construction in Korean. The Linguistic Association of Korean Journal 17.4, 1-23.

Heim, Irene. (1982). On the semantics of definite and indefinite noun phrases. Doctoral Dissertation, University of Massachusetts at Amherst.

Hornstein, Nobert. (2001). Move! A minimalist theory of construal. Oxford: Blackwell. Hornstein, Norbert, Howard Lasnik and Juan Uriagereka. (2003). The dynamics of islands: Speculations on the locality of movement. Linguistic Analysis 33, 149-175.

Kanyne, Richard. (1994). The antisymmetry of syntax. Cambridge, MA: MT Press. Kim, Alan Hyun-Ok. (1985). The grammar of focus in Korean syntax and its typological implications. Doctoral dissertation, University of Southern California. Ko, Heejeong. (2014). Types of right dislocation: Copies, adjuncts, and fragments in syntax. Paper presented at WAFL 11, University of York.

(2016). Gapless right-dislocation: The role of overt correlates. Language Research, 52.1, 3-32.

Lee, Jeong-Shik. (2009). A verb-initial single clause analysis for right-dislocated constructions in Korean. Studies in Modern Grammar 57, 127-157.

(2010a). Some remarks on NPI licensing. Studies in Generative Grammar 20, 251-275.

(2010b). Why only predicate-final in embedded clauses in Korean? Studies in Modern Grammar 61, 99-138.

(2010c). Island repair by pseduo-resumption. Studies in Generative Grammar 20.4, 655-670.

(2011a). Some loopholes of the double clause approach to the right dislocated construction in Korean. Studies in Modern Grammar 63, 113-146. (2011b). Layered VP and inner aspect. Studies in Modern Grammar 65, 45-72.

(2016a). Right dislocated elements and fragments in Korean. Studies in Generative Grammar 26, 115-141.

(2016b). A new look at syntactic structures in Korean: From the 
head-initial structural perspective. Seoul: HankookMunhwasa.

Lee, Jeong-Shik. (2017). Rethinking right-dislocation constructions. Studies in Generative Grammar 27, 1-36.

Lee, Wooseung. (2014). Argumental gaps in Korean. The Linguistic Association of Korean Journal 22, 1-29.

Park, Bum-Sik and Hyosik Kim. (2016). Case-drop, left-branch extraction and multiplicity in the right-dislocation construction. Linguistic Research 33.2, 259-297.

Park, Myung-Kwan and Ui-Jong Shin. (2016). [Ung 'yes'/Ani 'no', XP] involves right dislocation. Studies in Modern Grammar 91, 53-80.

Takano, Yuji. (2002). Surprising constituents. Journal of East Asian Linguistics 11, 243-301.

Tanaka, Hidekazu. (2001). Right-dislocation as scrambling. Journal of Linguistics 37, 551-579.

Jeong-Shik Lee

Department of English Language and Literature

Wonkwang University

460 Iksandae-ro, Iksan, Jeonbuk, 54538, Korea

E-mail: jslee@wonkwang.ac.kr

Received: February 27, 2017

Revised version received: April 15, 2017

Accepted: April 23, 2017 